\title{
A congruence-free semigroup associated with an infinite cardinal number
}

\author{
M. Paula O. Marques \\ Mathematical Institute, University of St. Andrews, Scotland \\ Centro de Matemática, Universidade do Minho, Portugal
}

\begin{abstract}
Let $X$ be a set with infinite cardinality $m$ and let $Q_{m}$ be the semigroup of balanced elements in $T(X)$, as described by Howie. If $I$ is the ideal $\left\{\alpha \in Q_{m}:|X \alpha|<m\right\}$ then the Rees factor $P_{m}=Q_{m} / I$ is 0 -bisimple and idempotent-generated. Its minimum non-trivial homomorphic image $P_{m}^{*}$ has both these properties and is congruence-free. Moreover, $P_{m}^{*}$ has depth 4 , in the sense that $\left[E\left(P_{m}^{*}\right)\right]^{4}=P_{m}^{*}$ and $\left[E\left(P_{m}^{*}\right)\right]^{3} \neq P_{m}^{*}$.
\end{abstract}

